komplementären Versorgungseinrichtungen gut ausgestattete städtische Region. Baden-Baden: Nomos.

Bildungswerk Irsee (2003, 2005 und 2007). Ambulante Sicherungsnachsorge. Möglichkeiten und Grenzen ambulanter forensischer Versorgung. Irsee: Jahresprogramm 2007.

Internetquelle: http://www.bildungswerkirsee.de.

BMJ (2006). Regierungsentwurf zur Reform der Führungsaufsicht. Internetquelle: http:// www.bmj.bund.de/files/1192/RegE _Fuehrungsaufsicht.pdf.

Auch Bundestagsdrucksache 256/06.

BMJ.Bund (2006). Pressemitteilung des Bundesjustizministeriums vom 05.April und 18.Mai 2006. Internetquelle: http:// www.bmj.bund.de.

BPTK - Bundespsychotherapeutenkammer (2006). Reform der Führungsaufsicht. 14.11.2006: Gesetz für entlassene Straftäter schießt über das Ziel hinaus. Internetquelle: http://www. bptk.de/show/321155.html.

DGSP, Deutsche Gesellschaft für soziale Psychiatrie (2006). Komplementäre Nachsorge für psychisch kranke Straftäter - Zertifikatskurs. Köln.
Egg, R. (Hg.) (2004). Ambulante Nachsorge nach Straf- und Maßregelvollzug. Wiesbaden: Schriftenreihe der Kriminologischen Zentralstelle, Bd. 44.

Freese, R. (2003). Ambulante Versorgung psychisch kranker Straftäter. Entstehung, Entwicklung, aktueller Stand und $\mathrm{Zu}$ kunft der 63er-Nachsorge in Hessen. Ein Nach-Lese-Buch. Lengerich: Pabst.

Hahn, G. (2003a). Resozialisierung psychisch kranker Straftäter. In: Cornel, H. et al. Handbuch der Resozialisierung. Baden-Baden: Nomos; S. 445-460. Hahn, G. (2003b). Sozialtherapie - Der Grundgedanke klinischer Sozialarbeit. Vortrag beim Fachtag „Sozialtherapie und Psychoedukation in der Klinischen Sozialarbeit" an der Fchochschule Coburg am 19.12.2003. Internetquelle: http://www.klinische-sozialarbeit.de/forschungundwissenschaft.

Hahn, G. (2005). Klinische Sozialarbeit in der forensischen Psychiatrie. Die Kunst der Zwangsbehandlung. Psychosozial, 29. Jg.. 2005, 101, Heft III; 67-73.

Hahn, G. (2007). Rückfallfreie Sexualstraftäter. Salutogenetische Faktoren bei ehemaligen
Maßregelpatienten. Bonn: Psychiatrie-Verlag.

Kurze, M. (1999). Soziale Arbeit und Strafjustiz. Wiesbaden: Schriftenreihe der Kriminologischen Zentralstelle, Bd. 26.

Kurze, M. (2004): Nachsorge und Bewährungshilfe/Führungsaufsicht. In: Egg (2004), S. 247266.

Nowara, S. (2001). Sexualstraftäter im Maßregelvollzug. Eine empirische Untersuchung $\mathrm{zu}$ Legalbewährung und kriminellen Karrieren. Wiesbaden: Schriftenreihe der Kriminologischen Zentralstelle, Bd. 32.

Pfäfflin, F. (2004). Sexualstraftaten. In: Venzlaff, U./Foerster, K. Psychiatrische Begutachtung. Ein praktisches Handbuch für Ärzte und Juristen. München: Urban \& Fischer.

Rauchfleisch, U. (1981). Dissozial. Entwicklung, Struktur und Psychodynamik dissozialer Persönlichkeiten. Göttingen: Vandenhoeck \& Ruprecht.

Rauchfleisch, U. (1999). Außenseiter der Gesellschaft. Psychodynamik und Möglichkeiten zur Psychotherapie Straffälliger. Göttingen: Vandenhoeck \& Ruprecht.

Schmidt-Quernheim, F. (2007). Kommunizierende Röhren Vom schwierigen Verhältnis zwischen Sozialpsychiatrie und Maßregelvollzug. Beobachtungen aus der Sicht einer forensischen Ambulanz. Psych. Prax. 2007, 34; 1-6.

Schmucker, M. (2004). Kann Therapie Rückfälle verhindern? Metaanalytische Befunde zur Wirksamkeit der Sexualstraftäterbehandlung. Herbolzheim: Centaurus.

Steinböck, H. et al. (2004). Ambulante Betreuung forensischer Patienten - vom Modell zur Institution. Recht \& Psychiatrie, 22 Jg., Heft 4; 199-207.

Stübner, S./Nedopil, N. (2004). Ambulante Sicherungsnachsorge des Bayerischen Staatsministeriums für Arbeit und Sozialordnung, Familie und Frauen Erste Ergebnisse aus der Begleitevaluation. In: Egg (2004), S. 143-168.

Volckart, B./Grünebaum, R. (2002). Maßregelvollzug. Das Recht des Vollzuges der Unterbringung nach $\S \S 63,64$ StGB in einem psychiatrischen Krankenhaus und in einer Entziehungsanstalt. Neuwied: Luchterhand.

Wagner, E./Werdenich, W. (Hg.) (1998). Forensische Psychotherapie. Psychotherapie im Zwangskontext von Justiz, Medizin und sozialer Kontrolle. Wien: Facultas.

\section{Evaluation des}

\section{Prostitutionsgesetzes}

Monika Frommel

Mit großer Verspätung veröffentlichte das Frauenministerium Anfang 2007 die Studie zu den Auswirkungen des Prostitutionsgesetzes. Die wichtigsten Kapitel sind überschrieben mit „Kriminalitätsbekämpfung und Prostitutionsgesetz" und auch die Vertiefung dieser Aspekte widmet sich vorrangig dem Ausstieg aus der Prostitution und der Frage, welche neuen und erweiterten Strafgesetze wohl sinnvoll sein könnten, um Jugendliche vor den
Gefahren dieser Branche zu bewahren. Eine der schnell umsetzbaren Konsequenzen wird wohl eine Angleichung der Schutzaltersgrenzen in den $\S \S 180 \mathrm{ff}$ StGB sein, zunächst einmal eine erweiterte Jugendschutznorm in $\S 182$ StGB. Dort soll die Schutzaltersgrenze 18 Jahre statt bisher 16 Jahre sein (BT Dr. 16/3439 vom 16.11.2006). Der Sache nach ist dies eine sehr weit gefasste Freierbestrafung ${ }^{1}$. Wegen des Verbots der Wohnungsgewäh- rung an unter 18 Jährige $\mathrm{zu}$ Zwecken der Prostitution finden sich junge Prostituiert vor allem auf dem Straßenstrich. Wer dort als Kunde nach Angeboten sucht, muss sich künftig vorsehen. Auch wer verdeckt agiert, geht ein Strafbarkeitsrisiko ein, da bei unter 18 Jährigen schon jeder Vorteil als Entgelt für sexuelle Handlungen gilt. Aber eine systematische Strafverfolgung ist eher nicht zu erwarten, sonst hätte in der Vergangenheit der Schutz der unter 16 Jährigen intensiver durchgesetzt werden müssen.

Die Studien sind sehr informativ. Aber das Ministerium hat alles dafür getan, dass es kein Gesamtkonzept zur Verbesserung der wirtschaftlichen Be- wegungsfreiheit erwachsener Prostituierter geben wird. Zwar wird lakonisch festgestellt, dass die bisherigen Maßnahmen auf Länderebene $\mathrm{zu}$ keiner verbesserten Umsetzung der zivilrechtlichen und verwaltungsrechtlichen Aspekte des ProstG geführt haben. Auch wird gut analysiert, dass und warum das ProstG nicht implementiert wurde. Auch die Notwendigkeit einer Verbesserung wird aufgezeigt. Aber letztlich münden die rechtspolitisch umsetzbaren Vorschläge ausschließlich in strafrechtliche Jugendschutznormen. Schutzaltersgrenzen besagen aber nicht einmal etwas über die tatsächlichen Angebote an junge Menschen in prekären Situationen und bei über 18 Jährigen bleibt es 
bei der fehlenden Regulierung2.

Auffallend ist die Zurückhaltung der Strafrechtswissenschaft. Zum Rechtsgut der §§ 180 a, 181 a StGB oder zum 2002 abgeschafften Unzuchtsdelikt der „Förderung der Prostitution" liest man fast nichts. Auch zum neu geschaffenen Delikt der ausbeuterischen Prostitution nach $\S 180$ a StGB schweigen sich die Kommentatoren aus. Dies ist einerseits unverständlich, da Rechtsgutsfragen ansonsten sehr beliebt sind. Andererseits ist es verständlich, da "Ausbeutung" normalerweise ein arbeits- oder zivilrechtliches Problem ist, das im Strafrecht lösen $\mathrm{zu}$ wollen, eher abwegig ist. Und auch der für das Täterstrafrecht der 1930er Jahre so typische Tätertyp des "Zuhälters" hat mit $\S 181$ a StGB aller liberalen Strafrechtsreformen zum Trotz überdauert und erfährt durch das ProstG lediglich einen Bedeutungswandel, denn die dirigistische Zuhälterei als solche ist seit 2002 erlaubt, auch wenn es gegenteilige, sich hartnäckig haltende Gerüchte gibt. Verboten sind nur ganz bestimmte Angriffe gegen die persönliche und wirtschaftliche Bewegungsfreiheit einer Prostituierten.

\section{Das Direktionsrecht der Ar- beitgeber in der Sex-Branche - eine lange strittige und nun geklärte Frage}

Eine Regelung wie in den Niederlanden, wo es üblich ist klare Verträge mit allen in Bordellen Tätigen zu schließen, scheiterte bis 2002 am Verdikt der Sittenwidrigkeit. Mittlerweile wären sie möglich. Die Gründe, wieso es in Deutschland noch immer so gut wie nie Arbeitsverträge in Bordelle gibt, haben also historische und kulturelle Gründe. Ein Rückblick in die jüngste Vergangenheit ergibt, dass schon vor dem ProstG nach der Rechtsprechung des Bundessozialgerichtes Prostituierte eigentlich steuerpflichtig gewesen wären. Auch gegen die So- zialversicherungspflicht hätte grundsätzlich nichts gesprochen (BSGE, Bd. 87, S. 53). Jedoch scheiterte vor 2002 die Einbeziehung Prostituierter in die Sozialversicherung faktisch daran, dass sich Bordellbetreiber durch die Meldung zur Sozialversicherung der Gefahr der Strafverfolgung nach $\S \S 180 \mathrm{a}$ Abs. 1 Nr. 2 StGB a. F. bzw. 181a Abs. 2 StGB a. F. (Förderung der Prostitution; Zuhälterei) ausgesetzt hätten. Unter anderem deshalb wurde 2002 sichergestellt, dass die Ausübung des in $\S 3$ ProstG festgehaltenen (eingeschränkten) Direktionsrechtes des Arbeitgebers für sich allein noch nicht zu einer Strafbarkeit nach den neu gefassten $\S \S 180 a$ Abs. 1, 181a Abs. 1 Nr. 2 führen kann.

Diese durch das ProstG diesbezüglich vorgegebenen Wertungen wurden auch zügig durch die höchstrichterliche Rechtsprechung ${ }^{3}$ umgesetzt. Dennoch herrschte eine echte oder nur vorgespielte Rechtsunsicherheit. Insbesondere die bayerischen Staatsanwaltschaften hielten Arbeitsverträge mit Prostituierten in Deutschland auch nach 2002 für verboten. Die StA Augsburg klagte deshalb in einem Musterprozess die Betreiber eines Bordells wegen dirigistischer Zuhälterei an, weil die Beschäftigten angewiesen worden waren im Saunabereich nackt zu bedienen. Spätestens mit dem spektakulären Nichteröffnungsbeschluss des Landgerichts Augsburg vom 1.09.2006 (zugunsten des Betriebs Collosseum) hat aber diese Auslegung ausgedient. Damit ist die gewünschte Rechtssicherheit geschaffen. Arbeitsverträge sind zulässig, dürfen aber die Prostituierten nicht knebeln und am Ausstieg hindern. Auch im übrigen ist die strafrechtliche Rechtsprechung so restriktiv geblieben wie vor 2002. So legt sie den Tatbestand der ausbeuterischen Prostitution nach $\S 180$ a StGB so eng aus wie früher die ausbeuterische $\mathrm{Zu}$ hälterei und bejaht „Ausbeutung" erst dann, wenn die Ar- beitsbedingungen so beschaffen sind, dass die Prostituierte nicht mehr aussteigen kann. Strafgerichte schützen also nicht die wirtschaftliche Bewegungsfreiheit der in Bordellen und auf dem Straßenstrich Beschäftigten, sondern orientieren sich an vermuteten Gefahren, die sich aus der Prostitution als solcher ergeben. Sie halten also letztlich nur das sexuelle Selbstbestimmungsrecht darüber zu entscheiden, ob man seine Arbeitskraft auf diese Weise anbiete, für entscheidend und nicht das Recht auf angemessene Arbeitsbedingungen. Deshalb stellen sie die wirtschaftliche Bewegungsfreiheit- trotz des Wortlauts der Vorschriften, der auf „Ausbeutung“ abstellt - ganz zurück und interpretieren die neu geschaffenen Strafnormen sehr restriktiv. Mit anderen Worten: wie das Preis-Leistungs-Verhältnis im einzelnen ausgestaltet ist, das überlässt die strafrechtliche Rechtsprechung der stummen Macht der Verhältnisse und diese könnten nur über gesellschaftliche Aktivitäten und einem verstärkten Zwang über die anderen Rechtsgebiete verändert werden ${ }^{4}$.

\section{Präventiver Rechtsschutz außerhalb des Strafrechts}

Es hätte nun nahe gelegen die Idee einer verwaltungs- und zivilrechtlichen Kontrolle der vorhandenen Bordelle aufzugreifen und aus der fehlenden IMpementierung Schlüsse $\mathrm{zu}$ ziehen. Dies ist nicht erfolgt. Verkannt wird nach wie vor die Bedeutung der $\S \S 180$ a, 181 a StGB als Verbotsnormen zur Kontrolle von zivilrechtlichen Verträgen (und damit auch faktischen Vertragsverhältnissen) über $\$ \S 134,242$ BGB. Nach der im Zivilrecht völlig unbestrittenen Rechtsprechung kommt es bei der Frage der Nichtigkeit von Verträgen nach § 134 BGB lediglich darauf an, ob der Sinn einer Verbotsnorm der Schutz einer Vertragspartei vor solchen Geschäften ist. Wenn das Verbot der Ausbeutung ei- nen Sinn haben soll, dann doch den Wuchermieten zu unterbinden. Wo die Grenze anzusetzen ist, kann nur Einzelfall bezogen mit Blick auf $\S 242$ BGB , also nicht pauschal, beantwortet werden, da jede Richtigkeitskontrolle nur konkret erfolgen kann. Aber im Gegensatz zum Strafrecht ist das Zivilrecht freier. Rechtsfortbildung ist möglich und das Analogieverbot hindert Zivilrichter nicht daran, ein entsprechendes Schutzrecht zu entwickeln. Unerlässlich ist jedenfalls ein effektiver Schutz der Prostituierten vor wucherischen Verträgen, und zwar nicht nur vor einer Ausbeutung i.S. einer gezielten Verarmung, sondern gegen alle Formen der krassen wirtschaftlichen Übervorteilung. Selbst wenn strafrechtlich eine rechtfertigende Einwilligung vorliegen sollte, dann schützt diese nur vor der Bestrafung, nicht aber vor einer zivilrechtlichen Rechtsfolge. Denn wenn Geschäfte dieser Art grob unbillig und tatbestandsmäßig i.S.d. $\S \S 180$ a, 181 a StGB sind, kommt es auf die Frage der Rechtfertigung weder für § 134 noch § 242 BGB nicht an. "Ausbeuter" müssen nicht strafbar handeln im Sinne einer rechtswidrig und schuldhaft begangenen Tat, sondern es genügt, wenn sie Forderungen durchsetzen wollen, welche krass unangemessen sind und deshalb einer Inhaltskontrolle unterzogen werden müssen. Die Prostituierte kann im übrigen nicht nur für die Zukunft, sondern auch rückwirkend erhebliche Summen zurückfordern bzw. die Finanzbehörde kann Bordellbesitzer zwingen, rückwirkend Steuern zu bezahlen. Schließlich kann auch rückwirkend verlangt werden Abgaben zu bezahlen. Alle entgegen stehenden Absprachen und Gepflogenheiten sind wegen § 134 BGB nichtig und über das Bereicherungsrecht rück abzuwickeln. Würden also die Finanzbehörden und würde die Arbeitsverwaltung kontrollieren und intervenieren, wäre die Lage erheblich besser, da 
die Nichtabführung von Steuern und Sozialabgaben nach $\S 266$ a StGB strafbar ist. Es könnte eine eindrucksvolle Drohkulisse aufgebaut werden statt den konturlosen „Menschenhandelsparagrafen" $\mathrm{zu}$ bemühen. Aber ohne Anstrengungen, die noch halblegal und illegal arbeitenden Bordelle in legale Dienstleistungszentren um $\mathrm{zu}$ bauen geht es nicht. Sowohl der Fiskus als auch die Sozialversicherungsanstalten, streng genommen müsste sie der Rechnungshof dazu verpflichten, zivil- und verwaltungsrechtliche Strategien - zusammen mit Beratungsstellen - aktiv zu unterstützen, also Runde Tische zu bilden, damit man sinnvoll und präventiv effektiv vorgehen kann. Erst ganz am Ende stünde dann das, was zurzeit als prima ratio empfohlen wird, die ausländerrechtliche Karte bei den illegal Beschäftigten. Wie immer man diese Karte ziehen möchte: sie hilft den illegal hier arbeitenden und um ihren Lohn betrogenen und erpressten Prostituierten am wenigsten, während zivilrechtlich operierende Beratungsstellen diesen Wirtschaftssektor langsam aber allmählich sanieren könnten. Die zurzeit besonders ausgegrenzten Migrantinnen finden spezialisierte Beratungsstellen vor und sollen dies auch in $\mathrm{Zu}$ kunft. Aber grundsätzlich sollten sich Beraterinnen von dem
Gedanken verabschieden, dass ihre Aufgabe primär eine psychosoziale sei und die beste Lösung der Ausstieg aus der Prostitution. Dies zu entscheiden ist Sache derer, die solche Dienstleistungen anbieten. Professionelle Beratung sollte gewerkschaftlich denken und effektive Wege der Verbesserung der Arbeitsbedingungen anbieten. Dies geht aber sicher nicht über Strafrecht pur, sondern allenfalls über $\S 134$ BGB i.V.m. den einschlägigen Strafnormen. Wo viel verdient wird, funktioniert eine zivilrechtliche Kontrolle jedenfalls besser abenteuerliche Konstruktionen wie Vermögensstrafen, Verfall und erweiterten Verfall in Strafverfahren, die letztlich ausländerrechtliche Ziele verfolgen.

- Die Lage aller Prostituierten kann nur durch eine konsequente Legalisierung durch Zivil-Arbeits-Gewerbe- und Bauordnungsrecht verbessert werden.

- Konsequente Strafverfolgung außerhalb der Jugendschutznormen setzt voraus, dass steuerrechtliche, gewerberechtliche, arbeits- und mietrechtliche Spielregeln verletzt worden sind. Dies setzt aber entsprechende Aktivitäten im Vorfeld der Strafbarkeit voraus.

- Die Betreiber eines Bordells und diejenigen, die einen
Straßenstrich organisieren, müssten sich den Regeln des sozialen Rechtsstaats unterwerfen. Nur dies könnte eine konsequente Disziplinierung kriminogener Männerbünde bewirken. Eine solche Kontrolle wäre mit Sicherheit den jetzigen Kriminalisierungsversuchen weit überlegen wäre.

\section{Fußnoten:}

1) Damit dürften Bestrebungen wie von der Bayerischen Staatsministerin der Justiz Beate Merk ZRP 8/2006, S. 250 - 252 vorgeschlagen erst einmal vom Tisch sein. Es wäre auch absurd gewesen, wenn die in $\S 232$ StGB verbotene Vermittlung in die Prostitution als „rechtswidrige Lage" definiert worden wäre, deren Missbrauch in einem neu $\mathrm{zu}$ schaffenden § 232 a StGB neu - Freierbestrafung - pönalisiert würde. Die Beispiele, welche Beate Merk in Fn. 3 ihres Textes gibt, betreffen ausländische Frauen, welche unter erkennbarem Zwang und unter unwürdigen Bedingungen zur Prostitution genötigt werden. Wer als Kunde sich solchen Situationen aussetzt, müsste eigentlich wegen gemeinschaftlicher Vergewaltigung bestraft werden nach nach § 177 Abs. 1 Nr. 3 (Ausnutzungsvariante) i.V.m. § 177 Abs. 2 Nr. 1 und 2 StGB. Wenn Staatsanwaltschaften trotz dieser klaren Rechtslage Strafverfahren einstellen, dann sollten die JustizministerInnen durch entsprechende Fortbildung und in Einzelfällen auch durch Weisungen einer solchen Praxis entgegen wirken statt weiteres Auffangstrafrecht $\mathrm{zu}$ schaffen.

2) Die systematisch völlig unverständliche Schutzaltersgrenze von 21 Jahren in $\S 232$ StGB haben Frommel/Schaar in NK 22005, S. 61 - 63 kritisiert. Vgl. ferner Thoma und Frommel wir im selben Heft der NK S. 52-61 zur Schwierigkeit Prostitution über Strafnormen regulieren zu wollen.

3) BGH NStZ-RR 2003, S. $361 \mathrm{zu}$ $\S 180 a$ Abs. 1 StGB; BGH NJW 2004, 81 zu $§ 181$ a Abs. 1 Nr. 2: Die bloße Vorgabe von festen Arbeitszeiten, Einsatzorten und Preisen ist noch kein „Bestimmen" im Sinne der Vorschrift; vgl. auch BayObLG StV 2004, S. 210.

4) Insoweit deskriptiv zutreffend das 2006 vom BMFSFJ veröffentliche Gutachten von Renzikowski „Reglementierung von Prostitution: Ziele und Probleme - eine kritische Betrachtung des ProstG. Seine abschließende Bewertung vertieft Aspekte des Jugendschutzes, regt aber keinen Rechtsprechungswandel bezüglich des Begriffs der „Ausbeutung" an, auch keine Stabilisierung der wirtschaftlichen Bewegungsfreiheit der in dieser Branche Arbeitenden. Auch die Anregungen des juristischen Kommentars von Margarete von Galen, Rechtsfragen der Prostitution 2004 werden innerhalb der Strafrechtswissenschaft nicht aufgegriffen, lediglich von Frommel, Kommentierung der $\S \S 180 \mathrm{ff}$ StGB im Nomos-Kommentar zum StGB, 2. Aufl. 2006.

\section{EU-UPDATE 1/2007}

Wolfgang Bogensberger

\section{Rechtsvorbereitung}

\section{Grünbuch über den diplomatischen und konsularischen Schutz des Unionsbürgers in Drittländern}

Jeder Unionsbürger genießt in einem Drittland, in dem der Mitgliedstaat, dessen Staatsangehörigkeit er besitzt, nicht vertreten ist, den diplomatischen und konsularischen Schutz eines jeden Mit- gliedstaats unter denselben Bedingungen wie Staatsangehörige dieses Staates. Dieser im Gemeinschaftsrecht (und in der Europäischen Grundrechtecharta) verankerte Schutz soll durch das am 28. November 2006 von der Kommission vorgelegte Grünbuch konkretisiert werden, etwa bei der Festnahme oder Haft eines Unionsbürgers in einem Nicht-EU-Land, bei schwerem Unfall oder schwerer Erkrankung, bei einem Gewaltverbrechen, bei einer Rückführung, in einer Notlage oder im Todesfall (herausragende Anlassfälle in letzter Zeit, bei welchen ganze Gruppen von Unionsbürgern in Schwierigkeiten geraten sind, waren etwa die Tsunami-Katastrophe Ende 2004 oder der Libanon-Krieg im Juli 2006).

Diesem Schutz kommt wachsende Bedeutung zu, weil Reisen von Unionsbürgern in Drittländer stark zunehmen (jährlich sind das rund 180 Millionen Reisen) und weil immer mehr Unionsbürger ihren Wohnsitz in solche Länder verlegen. Demgegenüber sind nur in 